\title{
SAÚDE MENTAL NA UNIVERSIDADE: A PERSPECTIVA DE UNIVERSITÁRIOS DA PERMANÊNCIA ESTUDANTIL
}

\author{
Luciana Marolla Garcia ${ }^{1}$, Vera Lucia Messias Fialho Capellini ${ }^{2}$, Verônica Lima dos Reis $^{3}$ \\ ${ }^{1}$ Doutoranda em Psicologia do Desenvolvimento e Aprendizagem pela Universidade Estadual Paulista - UNESP, Bauru, \\ SP. Vice-Coordenadora do Núcleo Técnico de Atenção Psicossocial (NTAPS) da UNESP, Bauru, SP. ORCID iD: \\ https://orcid.org/0000-0001-7625-4021 E-mail: lumarolla@yahoo.com \\ ${ }^{2}$ Doutora em Educação Especial pela Universidade Federal de São Carlos - UFSCar. Professa Adjunta do Departamento de \\ Educação, do Programa de Pós-graduação em Psicologia do Desenvolvimento e Aprendizagem e do Programa de Mestrado \\ Profissional em Docência para a Educação Básica, da Universidade Estadual Paulista - UNESP, Bauru, SP. Professora \\ Pesquisadora Produtividade (CNPQ). ORCID iD: https://orcid.org/0000-0002-9184-8319 E-mail:
} vera.capellini@unesp.br

${ }^{3}$ Pós-doutoranda no Programa de Pós-graduação em Psicologia do Desenvolvimento e Aprendizagem da Universidade Estadual Paulista - UNESP, Bauru, SP. É membro do Observatório Internacional de Inclusão, Interculturalidade e Inovação pedagógica (OIIIIPe), ambos cadastrados no CNPQ. ORCID iD: https://orcid.org/0000-0003-0681-0015. Email: veronica.reis@unesp.br

\section{RESUMO}

A forma como o aluno universitário se relaciona com a sociedade emocionalmente ou psicossocialmente vem ganhando destaque no mundo contemporâneo, o que tem sido ressaltado em pesquisas de universidades, assim como também tem levado as universidades a um olhar para a saúde mental e emocional dos seus alunos. O objetivo deste estudo foi analisar a opinião de alunos universitários da permanência estudantil sobre como a universidade poderia apoiá-los nas suas vivências psicossociais e saúde mental. Para tanto, o método, quali-quantitativo, envolveu a participação de 140 alunos, na faixa etária entre 17 e 24 anos, cadastrados no Núcleo Técnico de Atenção Psicossocial (NTAPS) da Unesp, Campus de Bauru, no ano de 2018, beneficiários de auxílio de permanência estudantil. O tratamento dos dados ocorreu por meio da análise de conteúdo textual das respostas dos alunos sobre como a universidade thes poderia apoiar em seus aspectos psicossociais. Esse conteúdo foi submetido ao software de estatística textual, Iramuteq, que por meio de duas técnicas de análise, apresentou uma nuvem de palavras e uma análise de similitude entre as palavras mais evocadas nos discursos dos alunos. A nuvem de palavras demonstrou graficamente que as palavras mais citadas foram "aluno", "universidade", "estar", "oferecer" e "atendimento psicológico" e a análise de similitude demonstrou as inter-relações entre estas palavras. Como resultado, obteve-se que os discursos dos alunos convergem para destacar a importância do atendimento psicológico durante o período da universidade, como parte do serviço de assistência estudantil.

Palavras-chave: Saúde mental. Universidade. Atendimento psicológico psicossocial. Permanência estudantil.

\section{MENTAL HEALTH AT THE UNIVERSITY: THE PERSPECTIVE OF UNIVERSITY STUDENTS}

\section{ABSTRACT}

The way in which university students relate to society emotionally or psychosocially has been gaining prominence in the contemporary world, which has been highlighted in university research, as well as leading universities to look at their students' mental and emotional health. . The aim of this study was to analyze the opinion of university students of the student stay on how the university could support them in their psychosocial and mental health experiences. To this end, the qualitative and quantitative method involved the participation of 140 students, aged between 17 and 24 years, registered at the Technical Center for Psychosocial Care (NTAPS) of Unesp, Campus de Bauru, in 2018, beneficiaries of student allowance. The treatment of the data occurred through the analysis of textual content of the students' 
answers about how the university could support them in their psychosocial aspects. This content was submitted to the textual statistics software, Iramuteq, which, through two analysis techniques, presented a word cloud and an analysis of similarity among the most evoked words in the students' speeches. The word cloud showed graphically that the most cited words were "student", "university", "being", "offering" and "psychological assistance" and the similarity analysis demonstrated the interrelationships between these words. As a result, it was obtained that the students' speeches converge to highlight the importance of psychological assistance during the university period, as part of the student assistance service.

Key words: Mental health. University. Psychological care. Psychosocial. Student assistance.

\section{SALUD MENTAL EN LA UNIVERSIDAD: LA PERSPECTIVA DE LOS ESTUDIANTES UNIVERSITARIOS}

\section{RESUMEN}

La forma en que los estudiantes universitarios se relacionan con la sociedad emocional o psicosocialmente ha ido ganando protagonismo en el mundo contemporáneo, lo que se ha destacado en la investigación universitaria, además de llevar a las universidades a mirar la salud mental y emocional de sus estudiantes. El objetivo de este estudio fue analizar la opinión de los estudiantes universitarios sobre la estancia de los estudiantes sobre cómo la universidad podría apoyarlos en sus experiencias psicosociales. Para ello, el método cualitativo y cuantitativo contó con la participación de 140 estudiantes, de entre 17 y 24 años, inscritos en el Centro Técnico de Atención Psicosocial (NTAPS) de la Unesp, Campus de Bauru, en 2018, beneficiarios de subsidio para estudiantes. El tratamiento de los datos se dio a través del análisis del contenido textual de las respuestas de los estudiantes sobre cómo la universidad podría apoyarlos en sus aspectos psicosociales. Este contenido fue sometido al software de estadística textual Iramuteq, el cual, mediante dos técnicas de análisis, presentó una nube de palabras y un análisis de similitud entre las palabras más evocadas en los discursos de los estudiantes. La nube de palabras mostró gráficamente que las palabras más citadas fueron "estudiante", "universidad", "ser", "ofrecer" y "asistencia psicológica" y el análisis de similitud demostró las interrelaciones entre estas palabras. Como resultado, se obtuvo que los discursos de los estudiantes converjan para resaltar la importancia de la asistencia psicológica durante el período universitario, como parte del servicio de asistencia al estudiante..

Palabras clave: Salud mental. Universidad. Atención psicológica. Psicosocial. Estancia estudiantil.

\section{INTRODUÇÃO}

Um crescimento acentuado nas pesquisas sobre prevenção em saúde mental tem ocorrido nas últimas décadas. Estima-se que a incidência de psicopatologias poderia ser reduzida por meio do incremento de fatores de proteção, como ações para melhoria da autoestima, rede de apoio, habilidades de enfrentamento, práticas de socialização positivas, engajamento social e a redução de estressores ambientais (ABREU; MIRANDA; MURTA, 2016).

A saúde mental tem por foco o desenvolvimento saudável do indivíduo nas suas diversas fases do ciclo de vida e busca reduzir, por ações antecipadas, os problemas e riscos que impedem tal desenvolvimento (O'CONNEL; BOAT; WARNER, 2009). Nessa direção, as intervenções preventivas buscam fortalecer os fatores de proteção.

No Brasil, as características dos programas preventivos em saúde mental e seus resultados são desconhecidos, como apontado por Abreu, Miranda e Murta (2016). A criação de novos mecanismos e investimentos públicos e privados para a disseminação de conhecimento e práticas vem sendo discutida e almeja-se que o conhecimento produzido nas universidades possa chegar à população (MARQUES, 2012).

Esses dados evidenciam que delineamentos adequados para estudos de avaliação de eficácia e efetividade de programas de prevenção, a partir de fatores de risco ou de transtornos em saúde mental ainda constituem uma necessidade do campo, como apontado há mais de uma década por Canoletti e Soares (2004) e Murta (2007).

As intervenções preventivas poderiam ser conduzidas ao longo do ciclo de vida, visto que transições de desenvolvimento humano necessitam de estratégias de enfrentamento para a adaptação a novas demandas que podem ser estressoras (FRAGELLI; GÜNTHER, 2008; XAVIER et. al., 2005). Poderiam ser realizadas em diferentes fases, por exemplo: na inserção de 
jovens no mercado profissional, na gestação do primeiro filho, no ingresso à faculdade, na transição para a aposentadoria, etc.

A fase da adolescência é caracterizada por inúmeras mudanças, considerando os aspectos biopsicossociais, que incluem a escolha profissional e preparação para o mercado de trabalho. No entanto, observa-se que o mercado tem se apresentado cada vez mais complexo, exigindo maior preparação para o início na atividade profissional. Neste contexto, o ingresso em um curso universitário tem sido apontado como uma opção inicial para o planejamento de carreira (BUENO; LEMOS; TOMÉ, 2004; HUTS; BARDAGI, 2006; VARGAS; ZAMPIERI, 2014). Entretanto, tal escolha não representa o fim dos conflitos relacionados ao futuro profissional, pois, o indivíduo encontra-se em constante desenvolvimento pessoal e acadêmico (BUENO; LEMOS; TOMÉ, 2004). A cada nova jornada na vida, os indivíduos vivenciam o processo de adaptação. Ao ingressar em uma universidade, é necessário passar por esse processo, pois os alunos se deparam com um sistema de ensino, regras institucionais, convívio social e dificuldades, geralmente diferentes dos que estavam habituados. Esse momento requer ajustamento e organização para experienciar as expectativas em relação aos domínios pessoais, acadêmicos, profissionais e sociais (OLIVEIRA; MORAIS, 2015). Tais situações constituem as vivências acadêmicas, cabendo às instituições de ensino superior a responsabilidade social de facilitar essa transição, por meio da criação de estratégias que visem a integração do indivíduo, para a sua permanência na universidade, com qualidade, até a conclusão do curso (OSSE; COSTA, 2011).

De acordo com Abreu, Miranda e Murta (2016) as intervenções preventivas e ações em saúde mental, têm como objetivo minimizar os fatores de risco e fortificar os fatores de proteção que inferem na saúde mental dos universitários.

Segundo Oliveira e Dias (2014) a adaptação acadêmica depende de uma série de fatores que fazem com que eles se sintam integrados ao curso e à universidade. Entretanto, esses aspectos não estão somente relacionados ao contexto acadêmico. $\mathrm{O}$ ajustamento ao ensino superior envolve o sentimento de pertencimento à turma e também o conhecimento das oportunidades oferecidas pela universidade como a rede de apoio. Além disso, grande parte dos estudantes lida com a separação da família. A saudade de casa (homesickness) tem sido identificada como uma consequência negativa da necessidade de se adaptar a um novo contexto, nesse caso, sair da casa dos pais para morar sozinho ou com outras pessoas (SCOPELLITI; TIBERIO, 2010). Estudantes que vivem sem a família podem apresentar níveis mais elevados de estresse do que estudantes que moram com os familiares (RULL et al., 2011), sendo mais comum encontrar entre os últimos, índices mais elevados de adaptação acadêmica (SOARES; GUISANDE; ALMEIDA, 2007). $O$ estresse acadêmico refere-se a uma reação adaptativa às demandas do meio que, quando prolongada, pode resultar em problemas, como desempenho acadêmico pobre, fracasso escolar, entre outros (RULL et al., 2011).

Dessa forma, é relevante a reflexão sobre o processo de ensino, principalmente na forma como as atividades acadêmicas são distribuídas ao longo dos cursos e a atenção para com o processo de adaptação dos estudantes na vida universitária. Daí a importância de serviços de apoio à saúde mental para dar suporte a esses casos, prevenindo o agravamento de sintomas e fortalecendo os mecanismos saudáveis e o potencial para lidar com os danos à saúde.

No Brasil, segundo dados do IBGE de 2019 , apenas $32,7 \%$ dos jovens entre 18 e 24 anos de idade, estão cursando o ensino superior. Um período de mais ou menos, quatro a seis anos, compõe o ciclo vital desses brasileiros. Esse período pode ser marcado por vivências individuais e coletivas que demandam, de quem experimenta essa fase, responsabilidade e sociabilidade. É um período em que ocorre, geralmente, o distanciamento familiar além de ser marcado por conflitos, decisões, escolhas e posturas importantes na trajetória de vida, pois também coincide na maioria das vezes com o início da fase adulta. Há estudos que revelam que esse pode ser um período ambíguo, de adoecimento mental, e até abuso de álcool e drogas (ASSIS; OLIVEIRA, 2010).

Resultado de pesquisa na Inglaterra, encontrou que calouros com problemas de saúde mental têm um desempenho acadêmico significativamente menor do que outros estudantes (BRUFFAERTS et al., 2018).

Peres, Santos e Coelho (2004) descrevem que os serviços de Clínicas-Escolas atualmente em universidades recebem estudantes que apresentam queixas, tanto de acolhimento e desabafo, quanto queixas de demanda para tratamento. $\mathrm{O}$ procedimento mais adotado 
nessas ocasiões é realizar uma primeira sessão e agendar um segundo atendimento na próxima semana. No segundo atendimento, o estagiário ou psicólogo responsável sugere o encaminhamento mais apropriado e verifica a disponibilidade do usuário em aderir aos serviços que podem ser psicoterapia individual ou grupal, encaminhamento para atendimento por profissionais da rede pública de saúde, profissionais conveniados que atuam em consultórios particulares ou para oficinas de convivência.

Estudantes universitários enfrentam problemas de saúde mental, como depressão, ansiedade e estresse, além de má qualidade do sono. Resultados de pesquisa com estudantes australianos mostram que os níveis de depressão, ansiedade e estresse estiveram significativamente associados à diminuição da qualidade do sono ou à diminuição da intenção de procurar ajuda. Uma análise de regressão múltipla previu que os estudantes eram mais propensos a relatar a intenção de procurar ajuda se tivessem menores escores de depressão e maiores escores de estresse. Níveis de intenção de busca de ajuda foram menores para homens do que para mulheres (ZOCHIL; THORSTEINSSON, 2018).

O desempenho no processo de aprendizagem envolve a inter-relação entre questões pessoais do estudante (fatores orgânicos, cognitivos, afetivos e motivacionais), aspectos relacionados à família e ao grupo social em que está inserido, e o ambiente escolar influenciado pelas interações, medidas pedagógicas e decretos governamentais que regem o ensino (SUHEIRO, 2006).

Nos Estados Unidos a preocupação e o interesse com a saúde mental do estudante universitário emergiram lentamente, porém, de maneira firme e constante, tendo como foco não somente a criação de cursos de higiene mental, mas também de programas de assistência psicológica e psiquiátrica. Por considerar que universitários podem passar por situações vulneráveis, estudos de 1910 denotam o surgimento de programas de prevenção de saúde mental em universitários, com objetivo de não permitir que estudantes capacitados desistissem de seus cursos ou que ficassem gravemente adoecidos (CERCHIARI; CAETANO; FACCENDA, 2005).

Em países europeus, como na Inglaterra, o primeiro centro de Saúde Estudantil surgiu em
1927, com um enfoque em doenças somáticas, que depois evoluiu para outros objetivos, devido ao número preocupante de suicídios entre os estudantes. Já na França, a preocupação com a saúde mental dos universitários iniciou por volta de 1950 e o foco de atuação naquele momento era lidar com abuso de substâncias como anfetaminas, o que depois transformou-se em serviço de assistência psicológica e psiquiátrica. Na Alemanha, também por volta da década de 1950, começaram a ser implantados serviços de saúde mental para universitários, como forma de maior atenção aos distúrbios psiquiátricos dos estudantes, corroborando com avanços nas áreas da Medicina e da Psicologia (CERCHIARI; CAETANO; FACCENDA, 2005).

Em 1956, em Nova Jersey, realizou-se a Primeira Conferência Internacional sobre Saúde Mental Estudantil, com especialistas de diversos países. O objetivo foi estabelecer intercâmbio entre os profissionais e criar programas que pudessem servir a todos países (LORETO, 1985).

No Brasil, em 1957 foi criado na Faculdade de Medicina da Universidade Federal de Pernambuco, junto à cadeira de Clínica Psiquiátrica, o primeiro Serviço de Higiene Mental e Psicologia Clínica, com a finalidade de oferecer assistência psicológica e psiquiátrica aos estudantes universitários, inicialmente, aos alunos de Medicina. E na década de 1960 surgiram novas iniciativas junto as Universidades Federais (LORETO, 1985).

Desde então, tem crescido o número de Instituições de Ensino Superior (IES) preocupadas com a saúde mental do estudante universitário e programas têm sido implantados nas universidades públicas brasileiras. Em São Paulo há o Serviço de Saúde Mental Escolar, da Escola Paulista de Medicina, que foi um dos pioneiros. A partir dos anos 1960, universidades de Minas Gerais, Rio de Janeiro e Rio Grande do Sul, também iniciaram serviços de Saúde Mental destinados a seus universitários. Apesar destas iniciativas e serviços que surgiram de longa data no Brasil, o número de pesquisas sobre a saúde mental do brasileiro ainda é escasso (CERCHIARI; CAETANO; FACCENDA, 2005).

A Universidade Estadual Paulista (Campus de Bauru) implantou o Núcleo Técnico de Atenção Psicossocial (NTAPS) em 2018 por meio da disponibilização de atendimento psicossocial aos discentes das três faculdades do campus (Faculdade de Ciências, Faculdade de Engenharia e Faculdade de Arquitetura, Artes e 
Comunicação). No primeiro ano (2018), o núcleo foi composto por duas Psicólogas, uma Assistente Social, oito estagiários de Psicologia e duas estagiárias de Serviço Social, ofertando um total de 82 horas semanais para atendimento. Em 2019, a equipe foi ampliada, e o Núcleo foi subdividido em três setores: Prevenção responsável por organizar, quinzenalmente, eventos de prevenção e promoção de saúde mental; Acolhimento - responsável pela entrevista inicial dos estudantes e pelo acolhimento com algumas sessões de atendimento psicológico; e por último a Assistência - responsável pelo atendimento psicológico de longo prazo. Atualmente, o serviço funciona de forma que todos os estudantes podem se inscrever por meio de um formulário de inscrição e, assim são agendados para entrevista inicial. O NTAPS recebe recursos das três unidades acima citadas e também de editais, parcerias e convênios.

Considerando a relevância do
desenvolvimento humano no período universitário e também a evolução da implantação de serviços de saúde mental para universitários, este estudo objetivou analisar a opinião de estudantes universitários da permanência estudantil sobre como a universidade poderia apoiá-los nas vivências psicossociais e saúde mental.

\section{DELINEAMENTO METODOLÓGICO}

Este estudo tem como amostra 140 alunos participantes de diferentes cursos de Graduação do Campus da UNESP de Bauru. Os dados foram coletados a partir de um questionário - aplicado pela equipe do Serviço Social da Unesp, equipe responsável, no momento, pela entrevista inicial dos participantes- composto de questões fechadas (que versavam sobre dados sociodemográficos) e uma questão aberta que indagava " $\mathrm{Na}$ sua opinião, de que maneira a faculdade poderia apoiar mais seus alunos com relação aos aspectos psicossociais?". Os dados referentes às questões fechadas são apresentados de modo a caracterizar a amostra e a questão aberta foi submetida ao software IRAMUTEQ para a análise textual do relato dos participantes.

O IRAMUTEQ foi elegido como recurso para a análise textual do relato dos participantes.
Trata-se de um software gratuito, desenvolvido sob a lógica do open source. Ele ancora-se no ambiente estatístico do software $R$ e na linguagem python (www.python.org). Este programa informático viabiliza diferentes tipos de análise de dados textuais, desde aquelas bem simples, como a lexicografa básica (cálculo de frequência de palavras), até análises multivariadas (classificação hierárquica descendente, análises de similitude). Ele organiza a distribuição do vocabulário de forma facilmente compreensível e visualmente clara por meio de funções estatísticas como, por exemplo, a análise de similitude e a nuvem de palavras.

Segundo Camargo e Justo (2013), no Brasil, desde a década de 1990, são utilizados alguns softwares para análises de textos, os quais, ao organizarem os dados, facilitam a realização de análises de conteúdo.

As respostas dos alunos foram divididas em 89 corpus textuais, cada qual refere-se a um segmento de texto. A análise de similitude baseia-se na teoria de grafos, o que possibilita identificar as ocorrências entre as palavras e seu resultado, com indicações da conexão entre as palavras, auxiliando na identificação da estrutura de um corpus textual, distinguindo também as partes comuns e as especificidades em função das variáveis ilustrativas (descritivas) identificadas na análise (MARCHAND; RATINAUD, 2012).

A nuvem de palavras as agrupa e as organiza graficamente em função da sua frequência. É uma análise lexical simples, porém graficamente interessante, na medida em que possibilita rápida identificação das palavras-chave de um corpus. Essas análises podem ser realizadas tanto a partir de um grupo de textos a respeito de uma determinada temática (corpus) reunidos em um único arquivo de texto; como a partir de tabelas ou planilhas, com linhas e colunas como no caso dos bancos de dados construídos a partir de testes de evocações livres.

\section{RESULTADOS E DISCUSSÃO}

Participaram deste estudo 140 alunos, 78 do gênero feminino e 62 do gênero masculino com idades entre 17 e 24 anos, de diferentes cursos (Figura 1). 
Figura 1. Curso de graduação dos participantes.

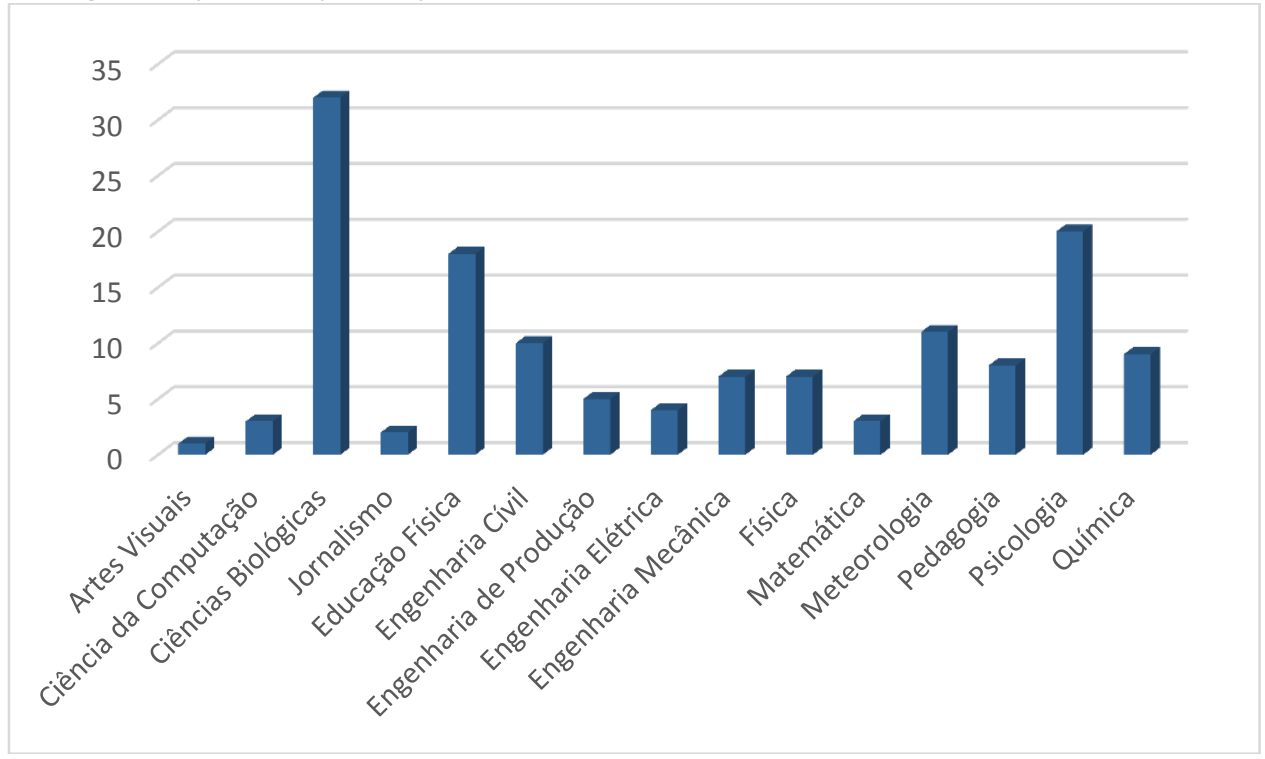

Fonte: As autoras.

Todos recebem algum auxílio financeiro: socioeconômico $(56,4 \%)$, aluguel $(37,9 \%)$ e moradia estudantil (5,7\%). Uma parte (15\%) recebe ainda bolsa de iniciação científica (CAPES, FAPESP, CNPq, PROEX). A maioria $(82,1 \%)$ relata que estudou em escola pública durante o ensino médio, contra 17,9\% que estudou em escola particular. Apenas 16 residem em Bauru com sua família, ou seja, a maioria não reside com sua família na mesma cidade onde fica a universidade; $50 \%$ desses alunos estudam em período integral; $48,6 \%$ estudam no horário noturno e apenas $1,4 \%$ estuda no período matutino.

Questionados sobre o grau de importância do acompanhamento psicossocial na universidade, $135(96,4 \%)$ responderam que é importante, 4 (2,9\%) que é indiferente e $1(0,7 \%)$ disse ser desnecessário, sendo que $52(37,1 \%)$ deles fizeram ou fazem tratamento psicológico. Todos utilizam algum tipo de recurso público socioassistenciais

(Figura 2).

Figura 2. Recursos públicos socioassistenciais utilizados pelos participantes.

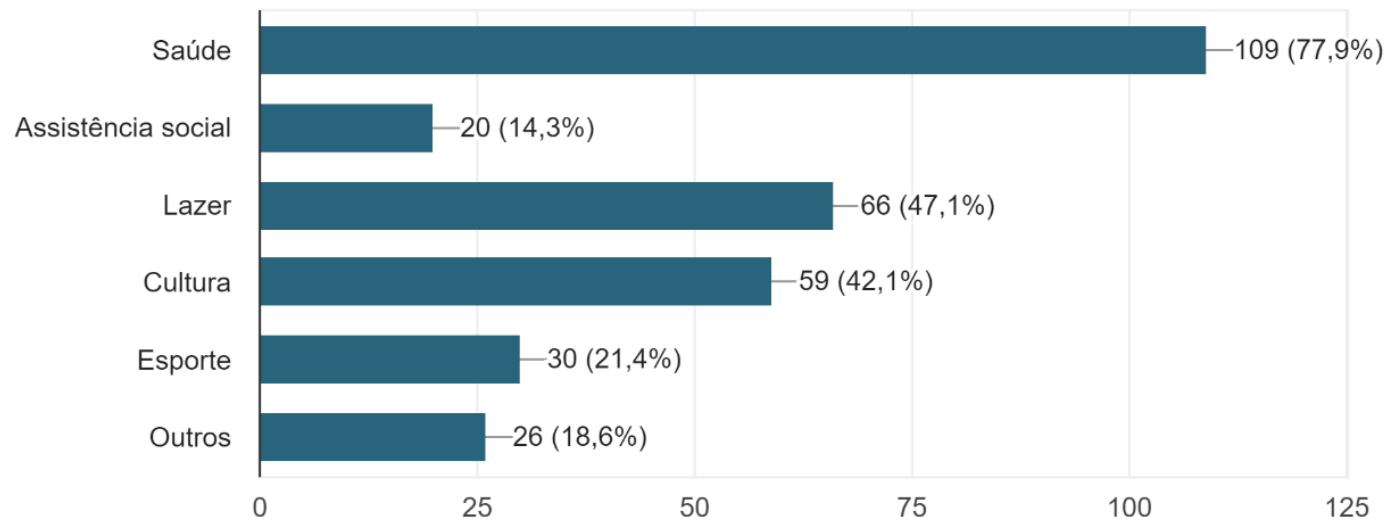

Fonte: As autoras.

Os participantes foram ainda inqueridos sobre os desafios do ambiente universitário (Figura 3), sendo que para 133 (95\%) deles manter-se financeiramente é a maior dificuldade. 
Figura 3. Desafios enfrentados pelos participantes na universidade.

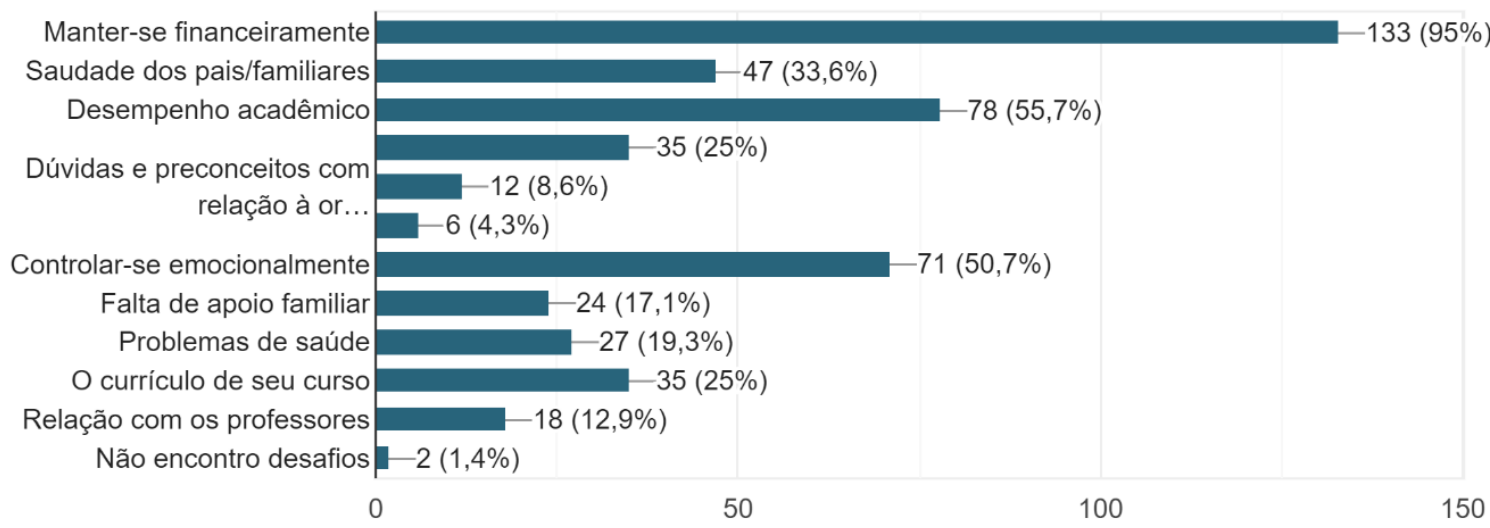

Fonte: As autoras.

Sobre a questão aberta ("De que maneira a faculdade poderia apoiar mais seus alunos com relação aos aspectos psicossociais?") foram criados 89 segmentos de texto a partir das respostas dos participantes e obteve-se uma nuvem de palavras e uma análise de similitude desses discursos. A análise da nuvem de palavras resultou nas seguintes palavras mais evocadas, sendo estas a palavras mais citadas em todos os discursos: aluno, universidade, atendimento psicológico, estar e oferecer. Em menor quantidade, mas merecendo destaque, mostrou que para os participantes teve significância as palavras: psicologia, apoio, maior, sentir, social, conforme pode ser verificado na Figura 4.

Figura 4. Nuvem de Palavras

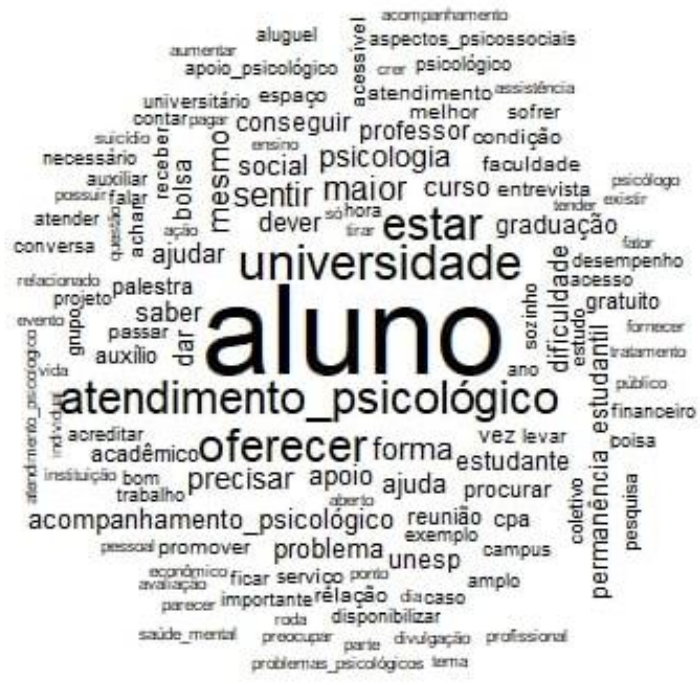

Fonte: Iramuteq (2019).

Já na Figura 5, podemos observar a análise de similitude criada pelo software, utilizando-se os mesmos 89 segmentos de textos extraídos do mesmo conteúdo do discurso dos participantes, sobre como esperam que a universidade poderia apoiá-los em seus aspectos psicossociais. 
Figura 5. Árvore de Palavras - Análise de Similitude

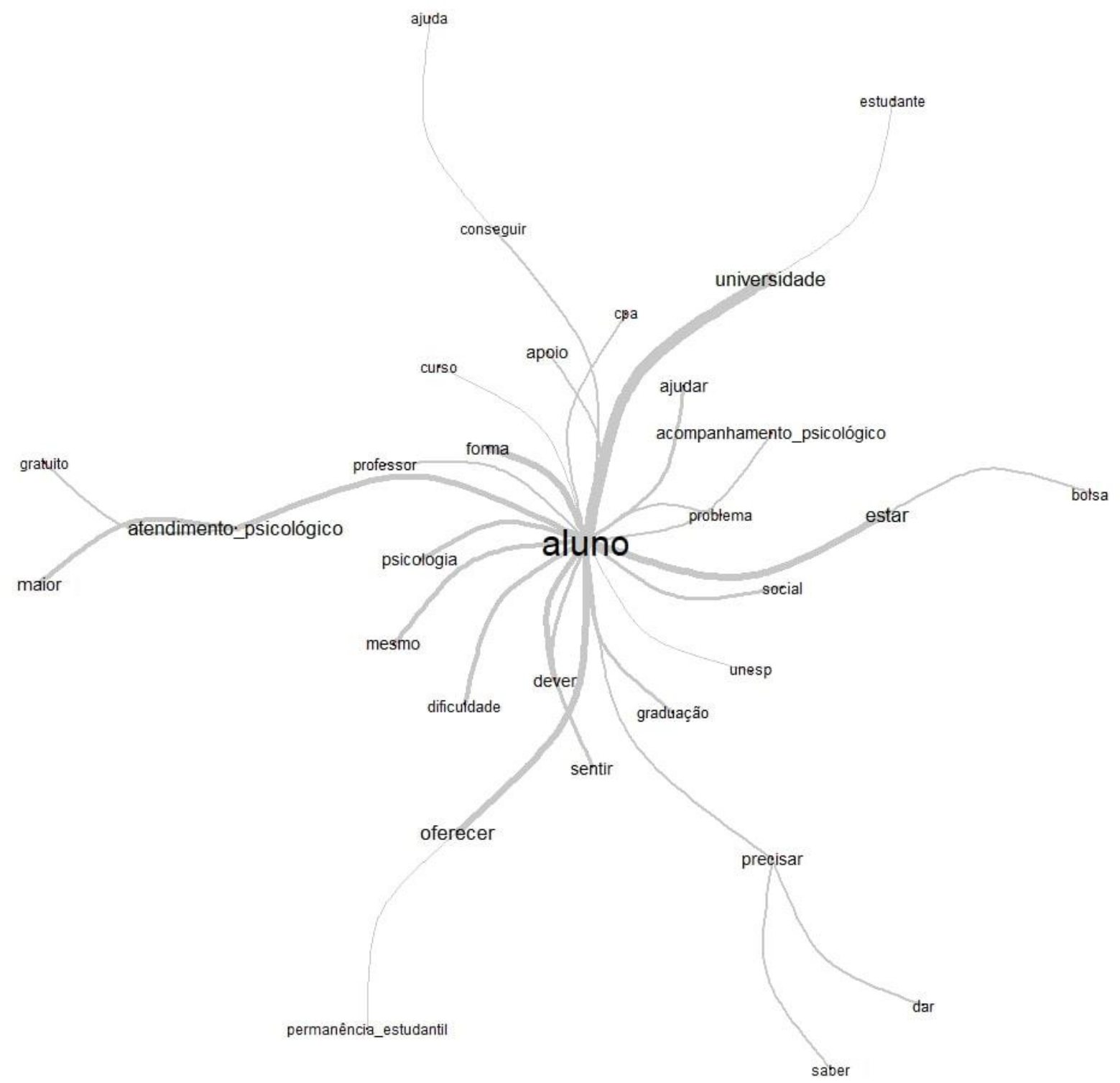

Fonte: Iramuteq (2019).

A análise de similitude identificou as ocorrências entre as palavras e as indicações da conexidade entre as palavras, auxiliando na identificação da estrutura de conteúdo de um corpus textual. Observa-se que a palavra aluno se destaca e dela se ramificam outras que apresentam expressão significativa como: atendimento psicológico, estar, oferecer, universidade, precisar. E em cada uma dessas há ramificações, por exemplo, na imagem está nítido como atendimento psicológico se relaciona com a palavra maior e gratuito. Da mesma forma, oferecer se ramifica em permanência estudantil, precisar se ramifica em dar e saber, conseguir se ramifica com ajuda.
Dentro do contexto universitário observouse que aparecem de forma relevante e repetida as interligações entre as palavras que foram mais citadas pelos participantes, ficando claro, quando expressam "atendimento psicológico" com interligação com "maior" e "gratuito".

Também houve outras ramificações da árvore, em que apareceu a palavra: acompanhamento psicológico, e outra ramificação em que apareceu a palavra "CPA", que, dentro do contexto dos alunos da Unesp de Bauru, significa Centro de Psicologia Aplicada, um dos locais no qual tanto os universitários quanto a comunidade externa podem receber atendimento psicológico na cidade 
de Bauru. E houve demais ramificações com a palavra "psicologia", inferindo-se que foi citada como fator importante a ser considerado pelos participantes.

Todas essas ramificações contêm apenas as palavras mais citadas nos discursos dos participantes, e não todas as palavras que foram citadas. Dado interessante, é que as palavras "atendimento psicológico", "CPA", "acompanhamento psicológico" e "psicologia", estão entre as palavras mais citadas em quatro diferentes formas. Também fica evidente a preocupação com as questões de "estar", e de "apoio", além da "permanência estudantil".

Pesquisa de Oliveira e Dias (2014) verificou que a rede de apoio dos estudantes entrevistados era composta, principalmente, por familiares e amigos. Os jovens afirmaram que a família, especialmente os genitores, ofereciam apoio emocional para lidar com 0 ingresso na universidade, como também com as dificuldades encontradas no curso escolhido e com a saída de casa, além de proporcionar suporte financeiro, quando necessário. Os estudantes entrevistados apreciavam o apoio recebido pelos pais. No caso deste estudo, os participantes, tendo ou não apoio familiar, esperam receber apoio ou criar uma rede de apoio dentro da universidade, assim como podemos observar através da palavra "apoio" que foi bastante mencionada pelos mesmos.

O apoio emocional dos pais ou demais familiares pode contribuir para o sentimento de segurança de forma que os jovens se sintam capazes de enfrentar a ansiedade envolvida nesta nova etapa da vida com nova integração social (TEIXEIRA; CASTRO; PICCOLO, 2007). Por outro lado, houve estudantes que negaram que a família tenha auxiliado na sua adaptação acadêmica. Infere-se que esses discentes acreditam que são os próprios jovens que precisam se ajustar à universidade e não parecem reconhecer o apoio dos familiares como importante para esse processo. Até porque, como mencionado anteriormente, muitos jovens estão fisicamente distantes de seus pais no momento da graduação (OLIVEIRA, 2010).

A percepção de uma vasta rede de apoio está associada com níveis mais elevados de adaptação acadêmica dos estudantes (FRIEDLANDER et al., 2007) e também com sua capacidade para fazer amizades, visto que os calouros consideram as amizades estabelecidas nas primeiras experiências universitárias como elementos que podem facilitar seu ajustamento ao ensino superior. Esse dado também corrobora com o apoio que os participantes esperam encontrar na universidade.

O apoio social constitui um elemento imprescindível para a integração dos estudantes à universidade. Contudo, nem sempre os discentes se sentem confortáveis para buscar ajuda, pois se preocupam em não sobrecarregar os outros com seus problemas (SMITH, 2014).

Figueiredo (2018) realizou pesquisa com 99 alunos universitários em Minas Gerais e concluiu que, a educação básica, embora tenha permitido o acesso ao curso superior, não habilitou os estudantes com competência ou conhecimentos necessários para adesão e permanência no ensino superior. Portanto, relatou que questões escolares, de educação básica, também podem se colocar como um obstáculo para o sucesso universitário. Nesse sentido, é necessária uma pedagogia numa perspectiva inclusiva que atenda às necessidades de todos os alunos universitários.

Entende-se que as necessidades relatadas pelos participantes nesta análise contextual corroboram com Assis e Oliveira (2010) quando ressaltam que os serviços de assistência estudantil ofertados por universidades refletem o compromisso social para com toda a comunidade. Compreende-se que ofertar serviços de assistência significa cuidar dos aspectos pessoais da vida dos estudantes do ponto de vista da saúde física e emocional, estudo e moradia, e ao mesmo tempo implementar políticas de assistência estudantil, respeitando e promulgando o direito à assistência e o compromisso social das universidades.

A saúde mental se tornou uma questão urgente nos campi universitários, à medida que os alunos procuram lidar com as muitas tensões da vida de estudante. A alta demanda por serviços também apresentou um desafio para os centros de aconselhamento universitário que muitas vezes têm longos tempos de espera para atender às necessidades de cada estudante (WILLIANS; CASE; ROBERT, 2018). A alta demanda de serviços, mencionada por estes autores está em consonância com o surgimento do NTAPS, que foi criado para atender alta demanda no campus.

Há o fato de que muitos estudantes com dificuldades de saúde mental têm maior probabilidade de abandonar a universidade, portanto, é vital que a saúde mental destes seja entendida para garantir que os mesmos tenham acesso ao apoio às suas necessidades durante o período universitário (CAGE; et al., 2020). De acordo com as palavras mencionadas na nuvem 
de palavras, como: apoio, oferecer, ajuda, podese inferir que os participantes esperam essa "ajuda" também como forma de evitar a evasão.

Ao considerar a palavra "apoio" e "estar" com interligação com a palavra "bolsa", pode-se inferir que os participantes esperam bolsa de estudos para estar, participar, frequentar a universidade. E neste eixo temático, podemos observar a interligação das palavras "oferecer" e "permanência estudantil".

Estudos revelam o aumento do número de alunos que tem acesso as universidades públicas, mas não tem condições sociais para permanência na universidade por quatro ou cinco anos, e muitas vezes desgastam-se em diversos tipos de empregos, dos quais tentam conciliar com seus estudos, mas que podem gerar prejuízos em suas atividades acadêmicas, sendo assim um desafio para a universidade pública a permanência dos alunos no período de sua graduação (COSTA; DIAS, 2015). Esse dado também corrobora com a opinião dos participantes sobre a importância da permanência estudantil.

Costa (2011) estudou as transformações pelas quais vem passando as universidades públicas e como isso interferiu na elaboração de políticas voltadas a permanência estudantil. Em seu estudo documental concluiu que a partir da década de 1990, houve maior espaço para discussão sobre o assunto e o desenvolvimento de alguns projetos que visam garantir a permanência dos estudantes na universidade. Contudo tais medidas ainda não são suficientes para garantir uma maior equidade na universidade. Para tanto, ainda se faz necessário maior investimento em recurso e indivíduos capacitados para gerir tais ações.

Quando o estudante decide evadir-se do curso, sem o ingresso prévio em outro, percebem-se sentimentos de medo de arrepender-se da decisão de não concluir uma graduação, de perder tempo e de não conseguir ingressar em outro curso superior, já que a permanência em uma graduação, mesmo que com insatisfação, parece garantir ao estudante $o$ ingresso no mercado de trabalho e sua ascensão social (BARLEM et al., 2012).

Por outro lado, aquele que conclui seu curso superior poderá estar habilitado a inserir-se em contextos de ensino, pesquisa e extensão, preparado para ingressar no mercado de trabalho (também porque já realizou os estágios obrigatórios), com visão e pensamento crítico-reflexivo, não somente para resolver os problemas cotidianos de sua área de formação, mas com condições de aplicar metodologias científicas em sua atuação, o que trará novos benefícios à sociedade, como o conhecimento produzido durante e após sua formação (CASTRO, 2002).

De acordo com Costa (2011, p. 2) "as políticas de assistência estudantis na educação superior têm a finalidade de destinar recursos e mecanismos para que os alunos possam permanecer na universidade e concluir seus estudos de modo eficaz".

Para tanto existe o Programa Nacional de Assistência Estudantil (PNAES), instituído em 2007, que é uma política do governo que tem como objetivo reduzir a evasão e o baixo desempenho em universidades. Estudos apontam que tanto a decisão de permanência no curso, quanto o desempenho e também o desenvolvimento psicossocial do aluno universitário, são afetados por fatores contextuais, pessoais, relacionais e acadêmicos (ANDRADE; TEIXEIRA, 2017).

Nascimento (2012) postula que há fragilidade sobre o que entendemos a respeito das necessidades estudantis. A autora questiona os conceitos de assistência e necessidade, afirmando, que é preciso entender que assistência não se reduz ao provimento de subsídios materiais, e necessidade está além do atendimento das necessidades de sobrevivência, dado que corrobora com outros estudos que demonstram que as necessidades dos alunos universitários não são apenas financeiras, e também corrobora com expressão de fala destes participantes no sentido de que a palavra apoio, também se refere a apoio social e não somente apoio ou auxílio financeiro. Assim, como quando não há apoio familiar financeiro para o estudante, a universidade oferece programas ou meios para prover esse auxílio, da mesma forma, quando não há apoio social ou emocional para o estudante, por parte da família, a universidade deve oferecer programas ou meios para isso.

Esse dado da assistência não somente monetária, como já mencionado anteriormente, também está de acordo com o discurso dos participantes desta pesquisa que citam que esperam receber apoio e permanência estudantil e ressaltam ainda a necessidade do acompanhamento psicológico durante seu percurso no transcorrer da formação acadêmica.

O impacto da saúde mental na educação também é um indicador significativo da procura de ajuda real, conforme os problemas de saúde mental começam a afetar o desempenho acadêmico, faz sentido que os alunos estejam mais inclinados a buscar ajuda. Essa descoberta também demonstra o impacto que as condições 
de saúde mental podem ter no funcionamento acadêmico como redução da concentração e da motivação, aumento da fadiga e isolamento social. Contudo esses dados demonstram também que é preciso ações para prevenir problemas de saúde mental como intervenções na melhoria da resiliência dos estudantes (CAGE et al., 2020).

Como ressaltam Leal et al. (2019), os estudantes enfrentam problemas emocionais, dificuldades financeiras, e inadaptação ao meio acadêmico. Além de afetar o desempenho acadêmico, estes aspectos podem estar direta ou indiretamente ligados ao desenvolvimento de problemas de saúde mental, inclusive depressão e suicídio. $O$ poder público e as instituições de ensino devem intervir, para enfrentar essas dificuldades e amenizar seus impactos na vida dos estudantes. Tais achados corroboram com os dados deste estudo de que a universidade deve intervir proporcionando apoio aos seus estudantes. Para estes autores, $42 \%$ dos universitários enfrentam dificuldades financeiras e a questão econômica interfere também no desempenho acadêmico, uma vez que, estes necessitam de aparato material e estrutural. São necessárias as aquisições de livros e materiais didáticos, além de recursos específicos para o curso, e também o acesso a computadores e internet. Esses dados também corroboram com a importância financeira da permanência estudantil, citada pelos participantes quando mencionam a palavra "bolsa".

Pesquisas recentes têm demonstrado também a preocupação com a saúde mental dos universitários que são minoria. Foram estudados o acesso a saúde mental dos estudantes negros (BANKS, 2020), os estudantes internacionais (WILLIANS; CASE; ROBERTS, 2018), estudantes nas perspectivas de gênero e orientação sexual (SANTOS; AZEVEDO; RAMOS, 2020) e a importância do apoio psicológico nestes casos. Esses dados corroboram com este estudo que identificou a opinião dos alunos de permanência estudantil que também podem ser considerado uma minoria e que também ressaltam a importância do apoio da universidade em sua adaptação.

Estudo de Banks (2020) na Universidade do Estado de Illions (EUA) encontrou resultados que também indicaram haver aumento nas atitudes em relação à busca de ajuda profissional pelos estudantes nos últimos anos e que os estudantes mais velhos eram mais propensos a concordar que buscar ajuda profissional pode ser benéfico. Dadas essas descobertas, a universidade em questão pôde promover a melhoria do acesso ao ambiente cultural e aos escritórios de recursos de aconselhamento. Assim, também, como nesse estudo, estes dados coletados em 2018, puderem subsidiar a nova estrutura do NTAPS, implementada em 2019 nos três setores de atuação, para que o núcleo seja um espaço de fala e de apoio, além de seus eventos preventivos.

\section{CONCLUSÕES}

O objetivo deste manuscrito foi analisar a opinião de alunos universitários da permanência estudantil sobre como a universidade poderia apoiá-los nas suas vivências psicossociais e saúde mental. Os resultados mostraram que na opinião dos participantes, eles esperam receber apoio psicossocial e atendimento psicológico.

Como limitações deste estudo observa-se que foram analisados apenas discursos de uma amostra de participantes de um projeto piloto de atenção psicossocial, em seu momento de implantação em uma universidade pública, por isso, a análise textual não pode apreender em sua totalidade como a universidade pode apoiar mais seus estudantes.

Dados ressaltados neste estudo são os de que é gritante o número de vezes que os estudantes mencionam as palavras "psicologia" ou "acompanhamento psicológico, ou "atendimento psicológico" como necessidade e algo que esperam da universidade de forma gratuita. Neste sentido, destaca-se a relevância de novas pesquisas na área de saúde mental e assistência estudantil para compreender de forma mais ampla como o atendimento psicológico poderá contribuir para o desenvolvimento do estudante em sua formação humana, acadêmica e psicossocial, dentro de uma política estudantil ou de saúde mental universitária.

Finalizando, resume-se que assistência estudantil, além de direito assegurado, pode contribuir na diminuição dos índices de evasão no ensino superior, refletindo no número de indivíduos e de famílias que deixam os estratos mais empobrecidos da hierarquia social e que, por meio do diploma, conquistam melhores empregos e melhor renda, revertendo o círculo de reprodução das desigualdades sociais existentes no Brasil. Sendo assim, ao garantir assistência socioeconômica a universitários, as 
universidades estão também cumprindo seu papel de socializar, ampliar e disseminar o conhecimento, e diminuir as desigualdades sociais (VARGAS, 2011).

\section{AGRADECIMENTOS}

Os autores declaram não haver qualquer potencial conflito de interesse que possa interferir na imparcialidade deste trabalho científico.

\section{REFERÊNCIAS}

ABREU, S.; MIRANDA, A. A. V.; MURTA, S. G. Programas Preventivos Brasileiros: Quem faz e como é feita a prevenção de saúde? Psico-USF, Bragança Paulista, v. 21, n.1, p. 163-177, 2016. Disponível

em:

https://www.scielo.br/scielo.php?script=sci artte xt\&pid=S1413-82712016000100163. Acesso em: 10 dez. 2020.

DOI:10.1590/1413-82712016210114

AMARGO, B. V.; JUSTO, A. M. Iramuteq: um software gratuito para análise de dados textuais. Temas em Psicologia, Ribeirão Preto, v. 21, n. 2, p. 513-518, 2013. Disponível em: http://pepsic.bvsalud.org/pdf/tp/v21n2/v21n2a1 6.pdf. Acesso em: 10 dez. 2020. DOI:10.9788/TP2013.2-16

ANDRADE, A. M. J.; TEIXEIRA, M. A. P. Áreas da política de assistência estudantil: relação com desempenho acadêmico, permanência e desenvolvimento psicossocial de universitários. Avaliação (Campinas), Sorocaba, v. 22, n. 2, p. 512-528, ago. 2017. Disponível em http://www.scielo.br/scielo.php?script=sci arttex t\&pid=S1414-

40772017000200512\&lng=pt\&nrm=iso. Acesso: 24 abr. 2019. DOI:10.1590/s141440772017000200014.

ASSIS, A. D.; OLIVEIRA, A. G. B. Vida universitária e saúde mental: atendimento às demandas de saúde e saúde mental de estudantes de uma universidade brasileira. Cadernos Brasileiros de Saúde Mental, Florianópolis, v. 2, n. 4-5, p. 159177, 2010.

BARLEM J. G. T. et al. Opção e evasão de um curso de graduação em enfermagem: percepção de estudantes evadidos. Rev Gaúcha Enferm., Porto Alegre (RS), v. 33, n. 2, p.132-138, 2012. Disponível https://www.scielo.br/scielo.php?script=sci artte xt\&pid=S1983-14472012000200019\&lng=en.

Acesso em: 10 dez. 2020.

DOI:10.1590/S1983-14472012000200019

BANKS, B. M. University Mental Health Outreach Targeting Students of Color, Journal of College Student Psychotherapy, v. 34, n. 1, p. 78-86, $2020 . \quad$ Disponível em: https://www.tandfonline.com/doi/abs/10.1080/8 7568225.2018.1539632? journalCode=wcsp20.

\begin{tabular}{llcl}
\hline Acesso em: & 10 & dez. \\
2020.DOI:10.1080/87568225.2018.1539632 &
\end{tabular}

BRUFFAERTS, R. et al. Mental health problems in college freshmen: Prevalence and academic functioning. Journal of Affective Disorders, v. 225, p. 97-103, 2018. Disponível em: https://www.sciencedirect.com/science/article/p ii/S0165032716324545. Acesso em: $10 \mathrm{dez}$. 2020. DOI:10.1016/i.jad.2017.07.044

BUENO, J. M. H; LEMOS, C. G.; TOMÉ, F. A. M. F. Interesses Profissionais de um grupo de estudantes de Psicologia e suas relações com inteligência e personalidade. Psicologia em Estudo, Maringá, v. 9, n. 2, p. 271-278, mai./ago, $2004 . \quad$ Disponível em: http://www.scielo.br/pdf/pe/v9n2/v9n2a13.pdf. Acesso em: 10 Jan. 2019. DOI:10.1590/S1413$\underline{73722004000200013}$

CAGE, E. et al. Barriers to accessing support for mental health issues at university. Studies in higher education, v. 45, n. 8, p. 1637-1649, 2020. Disponível em: https://www.tandfonline.com/doi/abs/10.1080/0 3075079.2018.1544237. Acesso em: $10 \mathrm{dez}$. 2020.DOI:10.1080/03075079.2018.1544237

CANOLETTI, B.; SOARES, C. B. Programas de prevenção ao consumo de drogas no Brasil: Uma análise da produção científica de 1991 a 2001. Interface - Comunicação, Saúde e Educação, Botucatu, n. 16, p. 115-129. 2004. Disponível em: http://www.scielo.br/pdf/icse/ v9n16/v9n16a10. Acesso em: 10 dez. 2020. DOI:10.1590/S1414$\underline{32832005000100010}$

CASTRO, C. A. A pesquisa discente nos cursos de graduação em biblioteconomia e ciência da informação. Transinformação, Campinas, v. 14, n. 1 p. 49-53, 2002. Disponível em: https://www.scielo.br/scielo.php?script=sci artte 
xt\&pid=S0103-37862002000100006. Acesso em: 10 dez. 2020. DOI:10.1590/S0103$\underline{37862002000100006}$

CERCHIARI, E. A. N.; CAETANO, D.; FACCENDA, O. Utilização do serviço de saúde mental em uma universidade pública. Psicologia Ciência e Profissão, Brasília-DF, v. 25, n. 2, p. 252-265, $2005 . \quad$ Disponível em: https://www.scielo.br/scielo.php?pid=S1414$98932005000200008 \&$ script $=s c i$ abstract $\&$ tlng $=p$ t. Acesso em: 10 dez. 2020. DOI:10.1590/S1414-98932005000200008

COSTA, S. L.; DIAS, S. M. B. A permanência no ensino superior e as estratégias institucionais de enfrentamento da evasão. Jornal de Políticas Educacionais, Curitiba, n. 9, v. 17, p. 51-60, 2015. Disponível em: https://revistas.ufpr.br/ipe/article/view/38650. Acesso em: 10 dez. 2020. DOI:10.5380/ipe.v9i17/18.38650

COSTA, S. G. A permanência na educação superior no brasil: uma análise das políticas de assistência estudantil. In: COLÓQUIO INTERNACIONAL SOBRE GESTÃO UNIVERSITÁRIA NA AMÉRICA DO SUL, 9., 2009, Florianópolis. Anais... Florianópolis: INPEAU/UFSC, 2009. p. 113.

FIGUEIREDO, A. C. Limites para afiliação à vida acadêmica de estudantes de camadas populares no contexto de expansão universitária. Educ. Pesquisa, São Paulo, v. 44, e173462, 2018. Disponível em: http://www.scielo.br/scielo.php?script=sci arttex t\&pid=S151797022018000100312\&lng=pt\&nrm=iso. Acesso: 26 abr. 2019. Epub 19-Jul-2018. DOI:10.1590/s1678-4634201844173462

FRAGELLI, T. B. O.; GÜNTHER, I. A. A promoção da saúde na perspectiva social ecológica. Revista Brasileira em Promoção da Saúde, Ceará, n. 21, v. 2, p. 151-158, 2008. Disponível em: https://periodicos.unifor.br/RBPS/article/view/67 4. Acesso em: $10 \mathrm{dez}$. 2020.DOI:10.5020/18061230.2008.p151

FRIEDLANDER, L. J. et al. Social support, selfesteem, and stress as predictors of adjustment to university among first-year undergraduates. Journal of College Student Development, n. 48, v. 3, p. 259-274, 2007. Disponível em: https://www.researchgate.net/publication/2367 48851 Social Support Self-

Esteem and Stress as Predictors of Adjustmen $\mathrm{t}$ to University Among First-

Year Undergraduates. Acesso em: 10 dez. 2020. DOI:10.1353/csd.2007.0024

GUERRA, L. C. B.; FERNANDES, A. S. A. O processo de Criação do Programa Universidade para Todos (PROUNI). Revista Política Hoje, Recife-PE, n. 2, v. 18, p. 281-305, 2009.

HUTS, C. S; BARDAGI, M. P. Indecisão profissional, ansiedade e depressão na adolescência: a influência dos estilos parentais. Psico-USF, Porto Alegre, v. 11, n. 1, p. 65-73, Jan./Jun, 2006. Disponível

em:

http://www.scielo.br/scielo.php?script=sci arttex t\&pid=S1413-82712006000100008. Acesso: 15 Jan. $2019 . \quad$ DOI:10.1590/S1413$\underline{82712006000100008}$

LEAL, K. S. et al. Desafios enfrentados na universidade pública e a saúde mental dos estudantes. Humanidades \& Inovação, PalmasTO, v. 8, n. 6, p. 59- 69, 2019.

LORETO, G. Contribuição do aconselhamento psicológico para a saúde mental dos universitários. Neurobiologia, Recife-PE, v. 28, p. 283-296, 1965.

MARQUES, F. Muito além das patentes. Pesquisa FAPESP, n. 197, p. 20-27, 2012. Disponível em: http://revistapesquisa.fapesp.br/2012/07/16/ muito-alem-das-patentes. Acesso em: $10 \mathrm{dez}$. 2020.

O'CONNEL, M. E.; BOAT, T.; WARNER, K. E. Preventing mental, emotional, and behavioral disorders among young people: Progress and possibilities. Washington, DC: The National Academies Press. 2009.

OLIVEIRA, C. T.; DIAS, A. C. C. Dificuldades na trajetória universitária e rede de apoio de calouros e formandos. PSICO, Porto Alegre, v. 45, n. 2, p. 187-197, abr./jun. 2014. Disponível em: https://revistaseletronicas.pucrs.br/ojs/index.ph $\mathrm{p} /$ revistapsico/article/view/13347. Acesso em: 10 dez. 2020. DOI:10.15448/19808623.2014.2.13347 
OLIVEIRA, R. E. C.; MORAIS, A. Vivências acadêmicas e adaptação de estudantes de uma universidade pública federal do Estado do Paraná. R. Educ. Públ., Cuiabá, v. 24, n. 57, p. 547-568, set./dez, 2015. Disponível em: http://periodicoscientificos.ufmt.br/ojs/index.ph p/educacaopublica/article/view/1796/pdf.

Acesso: 16 Jan. 2019.

OSSE, C. M. C.; COSTA, I. I. Saúde mental e qualidade de vida na moradia estudantil da Universidade de Brasília. Estudos de Psicologia, Campinas, v. 28, n. 1, p. 115-122, jan./mar., 2011. Disponível em: http://www.scielo.br/pdf/estpsi/v28n1/a12v28n 1.pdf. Acesso: 16 Jan. 2019. DOI:10.1590/S0103-166X2011000100012

PERES, R. S.; SANTOS, M. A.; COELHO, H. M. B. Perfil da clientela de um programa de prontoatendimento psicológico a estudantes universitários. Psicologia em Estudo, Maringá, v. 9, n. 1, p. 47-54, 2004. Disponível em: Acesso em: 10 dez. 2020.

DOI:10.1590/S1413-73722004000100007

RETINAUD, P.; MARCHAND, P. Application de la méthode ALCESTE aux corpus et stabilité des: analyse du avec IRAMUTEQ. In: JOURNÉES INTERNATIONALES D'ANALYSE STATISTIQUE DES DONNÉES TEXTUELLES, 11., 2012, Liége. Actes... Liége:Sorbonne, 2012. p. 835-844, 2012.

RULL, M. A. P. et al. Estrés académico en estudiantes universitarios. Psicología y Salud, Veracruz-MX, v. 21, n. 1, p. 31-37, 2011.

SANTOS, E. C.; AZEVEDO, H. V. P.; RAMOS, M. M. Preconceito e Saúde Mental: Estresse de Minoria em Jovens Universitários. Revista de Psicologia da IMED, Passo Fundo, v. 12, n. 2, p. 7-21, 2020. Disponível

em:

https://seer.imed.edu.br/index.php/revistapsico/ issue/view/180. Acesso em: 10 dez. 2020.

DOI:10.18256/2175-5027.2020.v12i2.3523

SCOPELLTI, M.; TIBERIO, L. Homesickness in university students: the role of multiple place attachment. Environment and behavior, n. 42, v. 3, p. 335-350, 2010. Disponível em: https://core.ac.uk/download/pdf/323214855.pdf - Acesso em: 10 dez. 2020.DOI:10.1177/0013916510361872
SMITH, M. E. Connecting students and families for support during the college transition. In: ACM CONFERENCE ON COMPUTER SUPPORTED COOPERATIVE WORK \& SOCIAL COMPUTING., 17, 2014. Proceedings... Baltimore, Maryland: Association for Computing Machinery, 2014. Disponível em: https://dl.acm.org/doi/10.1145/2556420.255683 2. Acesso em: 10 dez. 2020. DOI:10.1145/2556420.2556832

SOARES, A. P.; GUISANDE, M. A.; ALMEIDA, L. S. Autonomía y ajuste académico: un estudio con estudiantes portugueses de primer año. International Journal of Clinical and Health Psychology, n. 7, v. 3, p. 753-765, 2007.

SUEHIRO, A. C. B. Autoconceito e desempenho acadêmico em alunos de psicologia. Psicologia Argumento, Curitiba, v. 24, n. 44, p. 55-64, jan./mar. $2006 . \quad$ Disponível em: https://www.researchgate.net/publication/3768 6548 AUTOCONCEITO E DESEMPENHO ACADE MICO EM ALUNOS DE PSICOLOGIA. Acesso em: 10 dez. 2020. DOI:10.7213/rpa.v24i44.20135

TEIXEIRA, M. A. P.; CASTRO, G. D.; PICCOLO, L. R. Adaptação à universidade em estudantes universitários: um estudo correlacional. Interação em Psicologia, Curitiba, n. 11, v.2, p. 211-220, $2007 . \quad$ Disponível em: https://revistas.ufpr.br/psicologia/article/view/7 466. Acesso em: 10 dez. 2020.

DOI:10.5380/psi.v11i2.7466

VARGAS, J. M.; ZAMPIERI, A. C. B. A expectativas dos estudantes de Psicologia sobre a atuação profissional no mercado de trabalho. In: Mostra de Iniciação Científica Curso de Psicologia da FSG, 1., 2014, Caxias do Sul. Anais... Caxias do Sul:FSG, 2014. V.1, n,1, p.275-290. Disponível em: http://ojs.fsg.br/index.php/ampsic/article/view/1 402. Acesso: 16 Jan. 2019.

VARGAS, M. L. F., Educação superior, serviços de apoio aos estudantes e ao mercado de trabalho: um estudo com egressos da UFMG. Avaliação (Campinas), Sorocaba, v. 16, n. 1, p. 149-163, mar. 2011. Disponível em http://www.scielo.br/scielo.php?script=sci arttex t\&pid=S141440772011000100008\&lng=en\&nrm=iso. Acesso: 17 de maio de 2019. DOI:10.1590/S1414$\underline{40772011000100008}$ 
XAVIER, F. M. F. et al. Eldery people's definition of quality of life. Revista Brasileira de Psiquiatria, São Paulo, n. 25, v. 1, p. 31-39, 2003. Disponível em:

https://www.scielo.br/scielo.php?script=sci artte xt\&pid=S1516-44462003000100007. Acesso em: 10 dez. 2020.

DOI:10.1590/S1516-44462003000100007

WILLIANS, G.; CASE, R.; ROBERT, C. Understanding the Mental Health Issues of International Students on Campus. Educational Research: Theory and Practice, v. 29, n. 2, p. 1828, 2018. Disponível em: https://www.researchgate.net/publication/3299 14224 Teaching Design of Mental Health Educ ation Based on Case Teaching Method.

Acesso em: 10 dez. 2020.

DOI:10.12738/estp.2018.5.068

ZOCHIL, M. L.; THORSTEINSSON, E. B. Exploring poor sleep, mental health, and help-seeking intention in university students. Australian Journal of Psychology, v. 70, p. 41-47, 2018. Disponível em: https://aps.onlinelibrary.wiley.com/doi/full/10.1 111/ajpy.12160. Acesso em: 10 dez. 2020. DOI:10.1111/ajpy.12160 